\title{
Relationships between Cytauxzoon felis and African Piroplasmids
}

\author{
G. UILENBERG, F.F.J. FRANSSEN and N.M. PERIÉ \\ Department of Tropical Veterinary Medicine and Protozoology, Faculty of Veterinary \\ Medicine, University of Utrecht, P.O. Box 80.172, 3508 TD Utrecht (The Netherlands)
}

(Accepted for publication 30 October 1986)

\begin{abstract}
Uilenberg, G., Franssen, F.F.J. and Perié, N.M., 1987. Relationships between Cytauxzoon felis and African piroplasmids. Vet Parasitol , 26: 21-28.

A cat which had recovered from Cytauxzoon felis infection following treatment with the antitheilerial drug, parvaquone, showed an increase in piroplasm parasitemia after splenectomy and its blood was suitable for the preparation of antigen smears for the indirect fluorescent antibody test. High levels of antibodies were found in this cat after recovery, in two other cats sub-lethally infected with piroplasms and in sera of naturally infected bobcats.

Cats recovered from piroplasm infection died from cytauxzoonosis when challenged with organ material containing $C$. fels schizonts.

Tests with piroplasm antigens and positive sera of $C$ felis, South African Babesia felis and African Thelleria taurotragi showed no significant serological relationship between $C$ felis and the African parasites.

$C$ felss was not shown to be infective for splenectomized sheep.
\end{abstract}

\section{INTRODUCTION}

A newly discovered hemoprotozoan parasite was found to be the cause of fatal disease in domestic cats in the U.S.A. (Wagner, 1975, 1976). Because of the occurrence of exo-erythrocytic schizonts in macrophages and intra-erythrocytic piroplasms, it was assigned to the genus Cytauxzoon of the family Theileriidae and given the name Cytauxzoon felis by Kier (1979), whereas in the genus Theileria, schizonts are found in lymphocytes. The genus Cytauxzoon having been initially described in African ruminants (Neitz and Thomas, 1948; Neitz, 1957; Martin and Brocklesby, 1960; McCully et al., 1970), the possibility of an exotic threat to the U.S.A. was considered. However, it now appears that C. felis is a tick-transmitted parasite of the American bobcat ( Lynx rufus) (Glenn et al., 1983; Blouin et al., 1984). [ Interestingly, it had in all likelihood already been observed half a century ago (Wenyon and Hamerton, 1930).] 
Intriguing reports also suggest that the parasite might be infective and even pathogenic to non-felid animals (Ferris and Dardiri, 1978; Kier et al., 1982a) .

A serological test would be of value in epidemiological studies on C. fells in wild and domestic Felidae and other animals. Shindel et al. (1978) used serum from a recovered cat as antibody to demonstrate $C$. felis with an indirect fluorescent antibody test in frozen spleen sections of experimentally infected cats. However, preliminary results indicated that infected cats did not develop detectable antibodies before death, or only low titres at a late stage.

An African blood parasite of a wild Felis sp. in the Sudan, transmissible to domestic cats, was given the name Babesia felis by Davis (1929). A similar piroplasm of domestic cats in South Africa was called Nuttallia felis var. domestica (Jackson and Dunning, 1937), at present also designated as Babesia fells. The piroplasms are very similar to those of Theileria, as are those of $C$ felis and multiplication of the intra-erythrocytic piroplasms was reported to occur by quadruple division, as Maltese crosses, similar to that seen in C. felis (as a "diamond pattern") (Glenn et al., 1982) and in Theilerla (Uilenberg, 1965; Conrad et al., 1985) . It is so far unknown whether exo-erythrocytic schizonts, characteristic for the Theileriidae, occur in the life cycle of these African cat parasites. The genus name Nuttallia was given to species of Babesiidae which multiply by quadruple division, but is now usually synonymized with Babesia. It is, in any case, not valid for any of these blood parasites (Peirce, 1975 ). It should be noted that at least some "Nuttallia" species have lymphocytic schizont stages in the vertebrate host, such as Babesia equi (Schein et al., 1981 ) and B. microti (Mehlhorn and Schein, 1984) and may have to be transferred from the Babesiidae to the Theileriidae.

The purpose of the work reported here was to develop a serological test to demonstrate antibodies to $C$. felis and to use the test for a comparison of $C$. felis with South African B. felis and with Theileria taurotragi, generally assumed to be identical with a parasite originally described by Martin and Brocklesby (1960) as Cytauxzoon taurotragi (see Grootenhuis et al., 1979). It is a parasite of the African eland antelope (Taurotragus oryx) which also infects domestic cattle. An attempt was also made to verify the infectivity of $C$. felis for sheep, in view of the report by Kier et al. (1982a).

\section{MATERIALS AND METHODS}

Adult randomly bred cats, born in The Netherlands and two adult sheep of the Texel breed were used. One of the cats and both sheep were splenectomized prior to the experiments.

Deep-frozen stabilate of cat spleen containing schizonts of $C$. felis was received from the College of Veterinary Medicine, Columbia, MO, U.S.A. Frozen serum of a cat recovered from C. felis infection (Shindel et al., 1978) was obtained from the Plum Island Animal Disease Center, Greenport, NY, U.S.A. 
Frozen sera of bobcats, non-infected or naturally infected with $C$. felis, were sent from the College of Veterinary Medicine, Stillwater, OK, U.S.A. Deepfrozen antigen slides of $B$. felis, prepared from blood of infected cats and sera of cats, non-infected or infected with $B$. felis, were received from the Veterinary Research Institute, Onderstepoort, South Africa. For Theileria taurotragi, antigen slides and positive sera were made from blood of calves infected in our laboratory with the Tzaneen and Idobogo stocks (Uilenberg et al., 1982), fixed in acetone and stored at $-70^{\circ} \mathrm{C}$.

Spleen stabilate from Missouri was injected subcutaneously (s.c.) into a cat. The animal reacted with fever and was killed 12 days post infection (p.i.). Its spleen, containing numerous schizonts, was homogenized in phosphate-buffered saline, frozen slowly after dimethylsulphoxide had been added to a concentration of $10 \%$ and stored in liquid nitrogen as stabilate No. 153 . One milliliter aliquots of Stabilate 153 , thawed quickly in water at $37^{\circ} \mathrm{C}$, were used to infect or challenge the other experimental cats and one of the sheep, as indicated further on. Other animals were infected with fresh blood of parasitemic cats. Two of the cats were treated on the first day of fever with the drug parvaquone $\left(\right.$ Clexon $^{\mathrm{R}}$ ), used against cattle theileriosis (McHardy et al., 1983). Antigen smears were prepared from blood of a parasitemic cat (No. 8430) with a piroplasm parasitemia of $>1 \%$, fixed in acetone and stored at $-70^{\circ} \mathrm{C}$. Serum was prepared from all animals in the experiment at weekly intervals and stored at $-30^{\circ} \mathrm{C}$. Blood smears were made daily from animals in the experiment and stained with Giemsa, after methanol fixation.

The indirect fluorescent antibody test was carried out according to the method described by Brocklesby et al. (1971), with minor modifications. Serum dilutions were two-fold, starting at $1: 40$, to a maximum of $1: 20480$. Commercial rabbit anti-globulins conjugated with fluorescein isothiocyanate, directed against the animal species from which the sera were prepared, were used in the test, except in the case of bobcat sera, where anti-domestic cat globulins were used.

\section{RESULTS}

\section{Parvaquone treatment}

(i) Cat 8430 was infected s.c. with Stabilate 153 and reacted with fever, starting on Day 11 p.i. It was treated with $10 \mathrm{mg} \mathrm{kg}^{-1}$ of parvaquone intramuscularly (i.m.) on Day 11 p.i. and again on Day 13, this time with $16.5 \mathrm{mg}$ $\mathrm{kg}^{-1}$. It was severely affected clinically, but began to recover 6 days after the last treatment. It was challenged twice with Stabilate 153 (Fig. 1a) and proved solidly immune.

Very scanty piroplasms were seen in red cells on Day 19 p.i. and reappeared on Day 66. After splenectomy of the cat on Day 97, the parasitemia gradually 

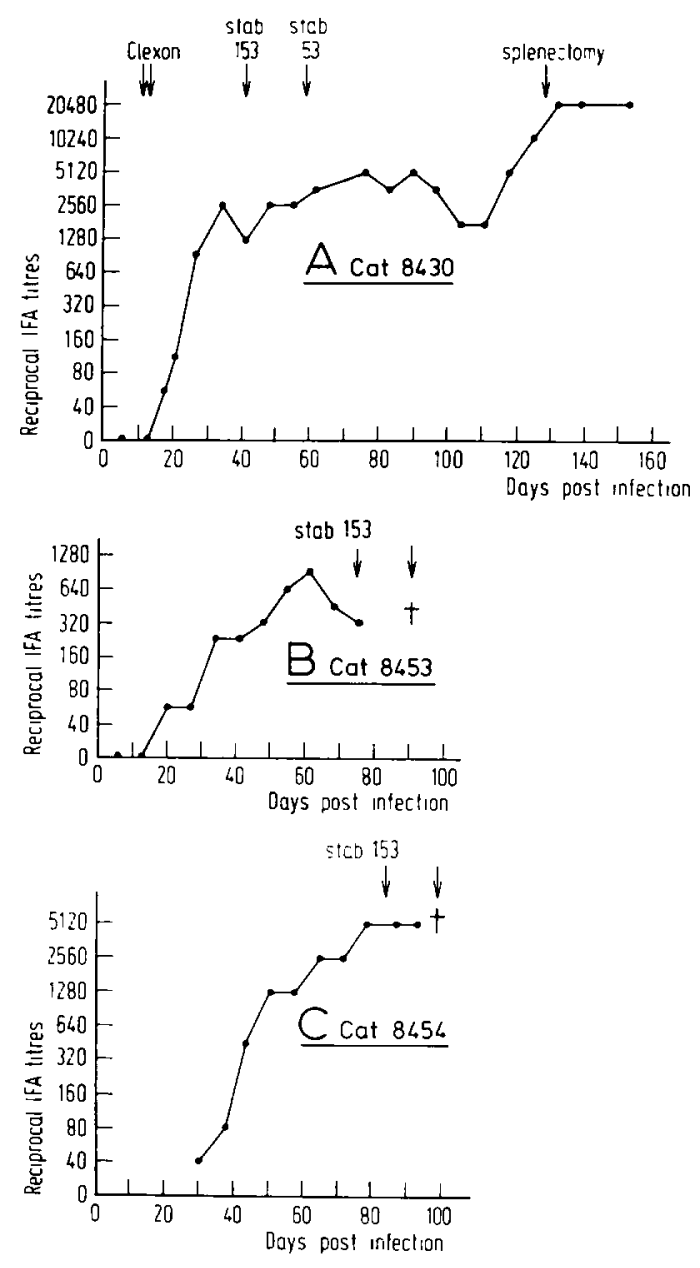

Fig. 1. Antibody response following serial testing of the sera of Cats 8430,8453 and 8454 with $C$ felis antigen.

increased until a maximum of $\sim 5 \%$ of red cells were infected between Days 122 and 130 p.i. No fever or clinical symptoms were associated with the parasitemia.

(ii) Cat 8429 was infected s.c. with Stabilate 153. It received parvaquone i.m. at $20 \mathrm{mg} \mathrm{kg}^{-1}$ on Day 10 p.i., when the thermal reaction started; the same treatment was repeated 2 days later. The animal appeared to recover initially, but relapsed on Day 19 p.i. and died 3 days later.

Transmission of piroplasms and antigenic relationship between piroplasms and schizonts

(i) Cat 8453 was injected intravenously (i.v.) with $2.5 \mathrm{ml}$ of fresh blood of Cat 8430 , containing scanty piroplasms. The cat had no fever reaction and 
showed no clinical signs of disease, although scanty piroplasms were seen irregularly in its blood between Days 10 and 73 p.i. The animal was challenged s.c. on Day 75 p.i. with $1 \mathrm{ml}$ of Stabilate 153. It reacted with fever 10 days later and died 16 days after the challenge (Fig. 1b). Schizonts were found in kidney, heart, liver and lung smears.

(ii) Cat 8454, splenectomized, received $5 \mathrm{ml}$ of fresh blood i.v. from Cat 8430 , containing scanty piroplasms. Piroplasms appeared 3 days p.i. and increased in numbers until $>50 \%$ of the erythrocytes were infected on Day 38 p.i. The high parasitemia persisted until Day 83 p.i. and was associated with anemia, but no other clinical signs of disease and no fever occurred. The cat was challenged s.c. with $1 \mathrm{ml}$ of Stabilate 153 on Day 84 p.i., reacted with fever 11 days later and died 15 days after the challenge (Fig. 1c). Schizonts were found in smears of the various organs.

Attempted transmission of C. felis to sheep

Splenectomized sheep No. 8441 received i.v. $5 \mathrm{ml}$ of fresh blood from Cat 8430 containing scanty piroplasms. Splenectomized sheep No. 8440 was injected s.c. with $1 \mathrm{ml}$ of Stabilate 153 and 3 months later i.v. with $5 \mathrm{ml}$ of fresh blood of Cat 8454 , which at that time had a parasitemia of $\sim 5 \%$. Neither sheep reacted clinically and no parasites were found in daily blood smears, made during a period of 35 days from Sheep 8441 and from Sheep 8440 during 5 months after the stabilate injection, 2 months after the fresh blood. Serial weekly sera of both sheep tested against $C$. felis antigen gave negative results (1:40) (during 1 month p.i. in Sheep 8441, 5 months in Sheep 8440).

Serological testing of cat and bobcat sera

Figures $1 a, b$ and $c$ show the results of serial testing of the sera of Cats 8430 , 8453 and 8454 with $C$. felis antigen. The results of testing various sera with $C$. felis and B. felis antigens are shown in Table I. No significant serological relationship was found between the two parasites.

Serological comparison of $\mathrm{C}$. felis and T. taurotragi

Table II shows the results of this comparison, which showed no serological relationship between the two parasites.

\section{DISCUSSION}

The indirect fluorescent antibody test, using piroplasm antigen of C. felis, can be used to detect antibodies to this parasite in recovered cats and could be useful for epidemiological studies in the natural host, the bobcat. Commercial 


\section{TABLE I}

Serological comparison of Cytauxzoon felıs and Babesıa felıs. Reciprocal IFA titres

\begin{tabular}{lll}
\hline & Antigens & \\
\cline { 2 - 3 } & C. felts & B fells \\
\hline Cat 8430, Day 6 p.i. & neg. & $40 / 80$ \\
Cat 8430, Day 90 p.i. & 5120 & $40 / 80$ \\
Bobcat 9 & neg. & neg. \\
Bobcat 10 & neg. & neg. \\
Bobcat 11 & 10240 & $80 / 160^{\mathrm{a}}$ \\
Bobcat 13 & 5120 & neg. \\
Bobcat 14 & neg. & neg \\
Cat Plum Island 00168 & $1280 / 2560$ & neg. \\
Cat 44 (S. Africa) & $40 / 80$ & $\geqslant 640$ (highest dilution) \\
Cat 45 (S. Africa) & neg. & 160 \\
Black cat (negative) (S. Africa) & neg. & neg \\
Black cat (positive) (S. Africa) & neg. & $160^{\mathrm{a}}$ \\
\hline
\end{tabular}

${ }^{a}$ Faint fluorescence.

neg. = negative.

anti-cat conjugated antibodies are apparently suitable for bobcat serum. The specificity of the test remains to be further explored, but is sufficient for distinguishing between American C. felis and South African B. felis, which do not appear to be closely related. No serological relationship could be detected between $C$ felis and T. taurotragl either.

Although Cat 8430 recovered after the administration of parvaquone, its case history and also the failure of parvaquone to cure Cat 8429 , show that it

\section{TABLE II}

Serological comparison of Cytauxzoon fells and Thellerıa taurotragl. Reciprocal IFA titres

\begin{tabular}{lll}
\hline Sera & Antigens & \\
\cline { 2 - 3 } & C felts & T taurotragı \\
\hline Cat 8430 & & \\
Pre-infection & $<40$ & 40 \\
62 days p.1. & $2560 / 5120$ & $40 / 80$ \\
160 days p.1. & 20480 & $40 / 80$ \\
Calf 278 & & \\
Pre-infection & $<40$ & 40 \\
31 days p.i. & $40 / 80$ & 1280 \\
38 days p.i. & n.d. & 5120 \\
73 days p.i. & $40 / 80$ & 1280 \\
\hline
\end{tabular}

n.d. $=$ not done. 
is unlikely that this drug will play any practical role in the treatment of cats suffering from cytauxzoonosis.

No evidence was obtained by parasitological and serological means of the infectivity of $C$. felis to sheep, contrary to reports by other workers (Kier et al., 1982a).

This study showed that piroplasms of $C$. felis are infective to cats, but do not cause fatal disease as they apparently do not transform to schizonts, the most pathogenic stage in the life cycle of the parasite. Piroplasms are also antigenically different from schizonts as they do not give protection to challenge with the latter. This conforms to similar observations made on other Theileriidae, recently summarized (Uilenberg et al., 1986) and also explains some of the results obtained with $C$. felis by others (Kier et al., 1982b).

\section{ACKNOWLEDGMENTS}

We are grateful to Dr. A.B. Kier.and Dr. L.G. Morehouse of Columbia, Dr. F.T. Potgieter of Onderstepoort, Dr. A. Kocan of Stillwater and Dr. D.H. Ferris of Greenport, for sending us the various materials. We thank Dr. M.J. Burridge, College of Veterinary Medicine, Gainesville, FL, U.S.A., for his encouragement and for his help in making personal contacts.

\section{REFERENCES}

Blouin, E.F., Kocan, A.A., Glenn, B.L. and Kocan, K.M., 1984. Transmission of Cytauxzoon fells Kier, 1979 from bobcats, Felts rufus (Schreber), to domestic cats by Dermacentor varlabllss (Say). J. Wildl. Dis., 20: 241-242

Brocklesby, D.W., Zwart, D. and Perié, N.M., 1971. Serological evidence for the identification of Babesia major in Britain. Res. Vet. Sc1., 12: 285-287.

Conrad, P.A., Kelly, B.G. and Brown, C.G.D., 1985. Intraerythrocytic schizogony of Theslerıa annulata. Parasitology, 91: 67-82.

Davis, L.J., 1929 On a piroplasm of the Sudanese wild cat (Felis ocreata). Trans. R. Soc. Trop. Med. Hyg., 22: 523-534.

Ferris, D.H. and Dardiri, A.H., 1978. Experimental infectıon of domestic animals with a newly discovered American feline protozoan. Abstracts of the 4th International Congress on Parasitology, Section C: 32 .

Glenn, B.L., Rolley, R.E. and Kocan, A.A., 1982. Cytauxzoon-like piroplasms in erythrocytes of wild-trapped bobcats in Oklahoma. J. Am. Vet. Med Assoc., 181: 1251-1253.

Glenn, B.L., Kocan, A.A. and Blouin, E.F., 1983. Cytauxzoonosis in bobcats. J. Am. Vet. Med. Assoc., 183: 1155-1158.

Grootenhu1s, J.G., Young, A.S., Dolan, T.T. and Stagg, D.A., 1979. Characteristics of Thelleria species (eland) infections in eland and cattle. Res. Vet. Sci., 27: 59-68.

Jackson, C. and Dunning, F.J., 1937. Biliary fever (nuttalliosis) of the cat. a case in the Stellenbosch District. J. S. Afr. Vet. Med. Assoc., 8: 83-88.

Kier, A.B., 1979. The etiology and pathogenesıs of feline cytauxzoonosis. Ph.D. Thesis, Columbia, 239 pp. 
Kier, A.B., Wightman, S.R. and Wagner, J.E., 1982a. Interspecies transmission of Cytauxzoon felts. Am. J. Vet. Res., 43. 102-105.

Kier, A.B., Wagner, J.E. and Morehouse, L G., 1982b. Experimental transmission of Cytauxzoon felts from bobcats (Lynx rufus) to domestic cats (Fells domestıcus). Am J. Vet. Res., 43 97-101

Martin, H. and Brocklesby, D.W., 1960. A new parasite of the eland Vet. Rec., 72: 331-332.

McCully, R.M., Keep, M.E. and Basson, P.A, 1970. Cytauxzoonosis in a giraffe (Giraffa camelopardalıs (Linnaeus, 1758)). Onderstepoort J. Vet. Res., 37: 7-10.

McHardy, N., Hudson, A.T., Morgan, D.W.T., Rae, D.G. and Dolan, T.T., 1983. Activity of 10 naphthoquinones, including parvaquone (993C) and menoctone, in cattle artificially infected with Thellerla parva. Res. Vet. Sci., 35: 347-352.

Mehlhorn, H. and Schein, E., 1984. The piroplasms: life cycle and sexual stages. Adv. Parasitol., $23 \cdot 37-103$

Neitz, W.O., 1957. Theileriosis, gonderioses and cytauxzoonoses: a review. Onderstepoort J. Vet. Res., 27: 275-430.

Neitz, W O. and Thomas, A.D., 1948. Cytauxzoon sylutcaprae gen. nov., a protozoon responsible for a hitherto undescribed disease in the duiker (Sylvıcapra grımmıa (Linné)). Onderstepoort J. Vet. Sci., 23. 63-76.

Peirce, M.A., 1975. Nuttallıa França, 1909 (Babesiidae) preoccupied by Nuttallıa Dall, 1898 (Psammobiidae ) : a reappraisal of the taxonomic position of the avian piroplasms. Int. J. Parasitol., 5: 285-287.

Schein, E., Rehbein, G., Voigt, W.P. and Zweygarth, E., 1981. Babesıa equi (Laveran 1901). 1. Development in horses and in lymphocyte culture. Tropenmed. Parasitol., 32: 223-237.

Shındel, N., Dardıri, A.H. and Ferris, D H., 1978. An indirect fluorescent antibody test for the detection of Cytauxzoon-like organisms in experimentally infected cats. Can. J. Comp. Med., 42: $460-465$.

Uılenberg, G., 1965. Acquisitions nouvelles dans la connaissance d'Haematoxenus veliferus hématozoaire des bovins à Madagascar. Bull. Soc. Pathol. Exot., 58: 432-445

Uilenberg, G., Perié, N.M., Lawrence, J.A., de Vos, A.J., Palıng, R.W. and Spanjer, A.A.M., 1982. Causal agents of bovine theilerıosis in southern Africa. Trop. Anim. Health Prod., 14: 127-140.

Uilenberg, G., Franssen, F.F.J. and Perié, N.M., 1986. Stage-specific antigenicity in Thellerıa annulata: a case report. Vet. Q., 8: 73-75.

Wagner, J.E., 1975. Cytauxzoonosis in domestic cats (Fells domestıca) in Missouri. J. Am. Vet. Med. Assoc., 167: 874.

Wagner, J.E, 1976. A fatal cytauxzoonosis-lıke disease in cats. J. Am. Vet. Med. Assoc., 168: 585-588.

Wenyon, C.M. and Hamerton, A.E., 1930. Puroplasms of the West African civet cat (Viverra ctvetta) and the Bay lynx (Felis rufa) of North America. Trans. R. Soc. Trop. Med. Hyg., 24: $7-8$. 